\title{
A SERIES AND ITS ASSOCIATED CONTINUED FRACTION ${ }^{1}$
}

\author{
J. L. DAVISON
}

ABSTRACT. Let $\alpha=(1+\sqrt{ } 5) / 2$. In this paper it is proved that

$$
T(\alpha) \equiv \sum_{r=1}^{\infty} \frac{1}{2^{[r \alpha]}}=\frac{1}{1+\frac{1}{2+\frac{1}{2+\frac{1}{4+\frac{1}{8+}}}}}
$$

where $t_{n}=2^{f_{n-2}}$ and $\left(f_{n}\right)$ is the Fibonacci sequence. It is also shown that $T(\alpha)$ is transcendental.

1. Introduction. If $x$ is a real number $\geqslant 1$ we may form the series $T(x)=$ $\sum_{r=1}^{\infty} 1 / 2^{[r x]}$. It is easy to see that the series is convergent and that $0<T(x)$ $\leqslant 1$.

In this paper we shall obtain a remarkable continued fraction expansion for $T(\alpha)$ where $\alpha=(1+\sqrt{ } 5) / 2$. Furthermore, we show that $T(\alpha)$ is transcendental.

Recall that the Fibonacci sequence $\left(f_{n}\right)$ can be defined by $f_{0}=1 ; f_{1}=1$; $f_{n}=f_{n-1}+f_{n-2}(n \geqslant 2)$ and that $\alpha$ has the continued fraction expansion [1, $1,1, \ldots]$ with convergents $f_{n} / f_{n-1}$ for $n \geqslant 1$. The notation and results on continued fractions used here are to be found in [2].

In $\$ 3$ we shall prove

TheOREM 1. $T(\alpha)=\left[0,1,2,2,4,8,32,256, \ldots, t_{n}, \ldots\right]$ where $t_{0}=1$, $t_{1}=1, t_{n}=2^{f_{n-2}}(n \geqslant 2)$.

2. Preliminary lemmas. Let $m$ be an arbitrary positive integer. Let $p=$ $f_{2 m-2}, q=f_{2 m-1}, p^{\prime}=f_{2 m}, q^{\prime}=f_{2 m+1}$. Then $q / p$ and $q^{\prime} / p^{\prime}$ are consecutive even convergents to $\alpha$. Clearly $p^{\prime}=p+q$ and $q^{\prime}=p^{\prime}+q$. The identities (i)

Received by the editors June 21, 1976.

AMS (MOS) subject classifications (1970). Primary 10F20, 10F35.

${ }^{1}$ This research was partially supported by NRC Grant 7703. 
$q^{\prime} p-p^{\prime} q=1$ and (ii) $q q^{\prime}=p^{\prime 2}-1$ are well known and can be easily proved by induction (on $m$ ).

For each integer $r \geqslant 0$ let $a_{r}=[r \alpha]$. The sequence begins as $1,3,4,6,8,9$, $11,12, \ldots$

LeMMA 1. With $p, q, p^{\prime}, q^{\prime}$ as defined above we have:

(a) $[r q / p]=a_{r}$ for $0 \leqslant r \leqslant p$,

(b) $\left[r q^{\prime} / p^{\prime}\right]=[r q / p]$ for $0 \leqslant r<p^{\prime}$.

Proof. (a) It is known that $0<\alpha-q / p<1 / p^{2}$. Thus $r q / p<r \alpha<r q / p$ $+r / p^{2} \leqslant(r q+1) / p$. If $r q / p<a_{r}$ then $r q<p a_{r}$ so that $r q+1 \leqslant p a_{r}$ and hence, $(r q+1) / p \leqslant a_{r}$. Then $a_{r}<r \alpha<(r q+1) / p \leqslant a_{r}$, which is a contradiction. Thus $[r q / p]=a_{r}$.

(b) Since $q^{\prime} / p^{\prime}=q / p+1 / p p^{\prime}$ it follows that $r q^{\prime} / p^{\prime}=r q / p+r / p p^{\prime}<$ $r q / p+1 / p$ if $0 \leqslant r<p^{\prime}$. By reasoning as in (a) we conclude that $\left[r q^{\prime} / p^{\prime}\right]=$ $[r q / p]$.

REMARK. Using (a) and (b) from Lemma 1, we can sharpen (a) to read: $[r q / p]=a_{r}$ for $0 \leqslant r<p^{\prime}$.

LeMma 2. With $p, q, p^{\prime}, q^{\prime}$ as defined above we have

(a) $a_{p+r}=a_{p}+a_{r}=q+a_{r}, 0 \leqslant r<q$,

(b) $a_{q+r}=p^{\prime}+a_{r}, 1 \leqslant r<p^{\prime}$.

Proof. (a) If $0 \leqslant r<q$ then $p+r<p+q=p^{\prime}$ so we may apply the Remark to conclude that $a_{p+r}=[(p+r) q / p]=[q+r q / p]=q+a_{r}$.

(b) Since $a_{q+r}=\left[(q+r) q^{\prime} / p^{\prime}\right]=\left[\left(q q^{\prime}+r q^{\prime}\right) / p^{\prime}\right]$ and $q q^{\prime}=p^{\prime 2}-1$, it follows that $a_{q+r}=p^{\prime}+\left[\left(r q^{\prime}-1\right) / p^{\prime}\right]$.

Note that this shows that $a_{q}=p^{\prime}-1$. Now $\left(p^{\prime}, q^{\prime}\right)=1$ so if $1 \leqslant r<p^{\prime}$, then $r q^{\prime} / p^{\prime}$ is not an integer and, hence, $\left[\left(r q^{\prime}-1\right) / p^{\prime}\right]=\left[r q^{\prime} / p^{\prime}\right]=a_{r}$. Thus $a_{q+r}=p^{\prime}+a_{r}$.

3. The main theorem. Let $S$ be the value of the continued fraction $[0,1,2,2$, $\left.4,8,32,256, \ldots, t_{n} \ldots\right]$ where $t_{0}=0, t_{1}=1$ and $t_{n}=2^{f_{n-2}}(n \geqslant 2)$. Denote the $n$th convergent to $S$ by $p_{n} / q_{n}$. Then it is clear that

$$
\begin{array}{ll}
p_{0}=0 ; p_{1}=1 ; p_{2}=2 ; p_{3}=5, \ldots, p_{n}=2^{f_{n-2}} p_{n-1}+p_{n-2} & (n \geqslant 2), \\
q_{0}=1 ; q_{1}=1 ; q_{2}=3 ; q_{3}=7, \ldots, q_{n}=2^{f_{n-2}} q_{n-1}+q_{n-2} & (n \geqslant 2) .
\end{array}
$$

The first six convergents to $S$ are $0,1, \frac{2}{3}, \frac{5}{7}, \frac{22}{31}, \frac{181}{255}$. It is easy to prove by induction that $q_{n}=2^{f_{n}}-1(n \geqslant 0)$. From general results on continued fractions we have

$$
p_{2 m-1} / q_{2 m-1}-p_{2 m+1} / q_{2 m+1}=2^{f_{2 m-1}} / q_{2 m-1} q_{2 m+1} .
$$

If $m, n$ are positive integers with $n \geqslant m$ then define

$$
T(m, n)=\sum_{r=1}^{\infty} \frac{1}{2^{[r n / m]}} .
$$

$T(m, n)$ is a rational number. By some computation it is found that $T(1,1)=1 ; T(2,3)=\frac{5}{7} ; T(5,8)=\frac{181}{255}$. These numbers occur as odd 
convergents to $S$. In fact, in general we have

LemMA 3. $T\left(f_{2 m}, f_{2 m+1}\right)=p_{2 m+1} / q_{2 m+1}$ for $m \geqslant 0$.

Proof. Since $T(1,1)=1=p_{1} / q_{1}$ it suffices to show that

$$
T\left(f_{2 m-2}, f_{2 m-1}\right)-T\left(f_{2 m}, f_{2 m+1}\right)=2^{f_{2 m-1}} /\left(2^{f_{2 m-1}}-1\right)\left(2^{f_{2 m+1}}-1\right)
$$

(this is the analogue of (1)).

$$
\begin{aligned}
& \text { Again let } p=f_{2 m-2}, q=f_{2 m-1}, p^{\prime}=f_{2 m} \text { and } q^{\prime}=f_{2 m+1} . \\
& \qquad \begin{aligned}
T(p, q)= & \sum_{r=1}^{\infty} \frac{1}{\left.2^{[r q / p}\right]}=\frac{1}{2^{a_{1}}}+\frac{1}{2^{a_{2}}}+\cdots+\frac{1}{2^{q}} \\
& +\frac{1}{2^{a_{1}+q}}+\cdots+\frac{1}{2^{2 q}}+\cdots \\
= & \left(1 / 2^{a_{1}}+1 / 2^{a_{2}}+\cdots+1 / 2^{q}\right)\left(2^{q} /\left(2^{q}-1\right)\right) \\
= & \left(2^{q-a_{1}}+\cdots+2^{q-a_{p-1}}+1\right) /\left(2^{q}-1\right) .
\end{aligned}
\end{aligned}
$$

Similarly

$$
T\left(p^{\prime}, q^{\prime}\right)=\frac{\left(2^{q^{\prime}-a_{1}}+\cdots+2^{q^{\prime}-a_{p^{\prime}-1}}+1\right)}{2^{q^{\prime}}-1} .
$$

Hence

$$
\begin{aligned}
& T(p, q)-T\left(p^{\prime}, q^{\prime}\right) \\
& \quad=\frac{\left[\left(2^{q^{\prime}}-1\right)\left(2^{q-a_{1}}+\cdots+2^{q-a_{p-1}}+1\right)-\left(2^{q}-1\right)\left(2^{q^{q}-a_{1}}+\cdots+2^{q^{\prime}-q}+\cdots+1\right)\right]}{\left(2^{q}-1\right)\left(2^{q^{\prime}}-1\right)} .
\end{aligned}
$$

The numerator simplifies to

$$
\begin{aligned}
-\left(2^{q-a_{1}}+2^{q-a_{2}}+\cdots+1\right)-\left(2^{q+q^{\prime}-a_{p+1}}\right. & \left.+\cdots+2^{q}\right) \\
& +\left(2^{q^{\prime}-a_{1}}+\cdots+1\right) .
\end{aligned}
$$

Now, by Lemma 2, $a_{p+r}=q+a_{r}$ and $a_{q+r}=p^{\prime}+a_{r}$. Thus we have $q+q^{\prime}$ $-a_{p+r}=q^{\prime}-a_{r}(0 \leqslant r<q)$ and $q-a_{r}=q^{\prime}-a_{p^{\prime}-p+r} \quad(1 \leqslant r \leqslant p)$. Hence the numerator becomes $-2^{q}+2^{q^{\prime}-a_{p^{\prime}-p}}=-2^{q}+2^{q+1}=2^{q}$ and $T(p, q)-T\left(p^{\prime}, q^{\prime}\right)=2^{q} /\left(2^{q}-1\right)\left(2^{q^{\prime}}-1\right)$.

Proof of Theorem 1. Lemma $1\left(\right.$ a) shows that $|T(\alpha)-T(p, q)|<1 / 2^{q}$ since both series agree up to and including the term $1 / 2^{q}$. Thus

$$
T\left(f_{2 m}, f_{2 m+1}\right) \rightarrow T(\alpha) \text { as } m \rightarrow \infty \text {. }
$$

But we have $p_{2 m+1} / q_{2 m+1} \rightarrow S$ as $m \rightarrow \infty$. By Lemma 3 , it follows that $S=T(\alpha)$.

THEOREM 2. $T(\alpha)$ is transcendental. In fact the inequality $|T(\alpha)-a / b|<$ $1 / b^{2.6}$ is true for an infinity of positive integer pairs $a, b$.

Proof. If $p, q$ are as in Lemma 3 then, using the Remark, it is easy to see that 


$$
0 \leqslant T(p, q)-T(\alpha) \leqslant\left(1 / 2^{q^{\prime}-1}+1 / 2^{q^{\prime}}+\ldots\right)=1 / 2^{q^{\prime}-2} .
$$

Now $T(p, q)=a / b$ where $b=2^{q}-1$. Also $q^{\prime} / q \rightarrow(3+\sqrt{ } 5) / 2$ as $m \rightarrow \infty$. Thus $q^{\prime}>2.6 q+2$ for large enough $q$ and, hence, $0<T(p, q)-T(\alpha)<$ $1 / b^{2.6}$. By Roth's Theorem ([1] or [3]) we conclude that $T(\alpha)$ is transcendental.

\section{REFERENCES}

1. J. W. S. Cassels, An introduction to Diophantine approximation, Cambridge Tracts in Math. and Math. Phys., no. 45, Cambridge Univ. Press, London and New York, 1957. MR 19, 396.

2. G. H. Hardy and E. M. Wright, An introduction to the theory of numbers, 3rd ed., Clarendon Press, Oxford, 1954. MR 16, 673.

3. K. F. Roth, Rational approximations to algebraic numbers, Mathematika 2 (1955), 1-20; Corrigendum, 168. MR 17, 242.

Department of Mathematics, Laurentian University, Sudbury, Ontario, Canada 